\title{
Time-sensitive Travel Route Recommendations
}

\author{
Huanliang Sun ${ }^{1, a^{*}}$, Dacheng Ye ${ }^{2, b}$ and Junling Liü
}

${ }^{2}$ School of Information and Control Engineering, Shenyang Jianzhu University, Shenyang 110168, China

a1968207285@qq.com, b571404720@qq.com, ' liuj|@sjzu.edu.cn

* The corresponding author

Keywords: Route recommendation; Time-sensitive; Transition graph model; Check-in data; Hierarchical clustering

\begin{abstract}
With the rapid development of mobile Internet and the widespread use of mobile devices, social networking platforms such as Gowalla and Foursquare provide sign-in sharing capabilities. They use these functions to collect a large number of check-in data that can reflect the user's travel patterns. These data can effectively support the recommendation and search of travel routes. This paper studies the recommendation of tourist routes for the change of scenic spots popularity caused by time change in spatial area. We quantitatively represent the changes in the popularity of the locations in the region in the form of time series, and recommend the travel routes that are most suitable for the travel time of the users. The route recommendation of the dynamic transition graph mode can be used for tourism route recommendation, scenic area construction, and safe facility deployment. The proposed dynamic transition graph model was constructed with real data sets and sufficient experiments were conducted. It is verified that under the condition of different parameters, the user's income in the recommended route is higher than the existing work by over $10 \%$. This verifies the accuracy and effectiveness of the proposed method.
\end{abstract}

\section{Introduction}

With the rapid development and popularization of smart phones, more people have access to social networks through smart phones. As the accuracy of the GPS global positioning system is gradually improved, people can use social networks to share their travel and location anywhere, anytime. However, huge data sharing, mass tourism information and reviews make it difficult for users to simply plan their own trips through data. With the rapid development of tourism, tourists have greatly improved their consumption concepts and consumption characteristics. They not only began to pursue the rationality of the route, but also pursued the individualization of the route. Therefore, the travel route recommendation has attracted people's extensive attention and pursuit. Visitors will consider more and more tourism factors in their own trips, so that they can get a better travel experience. Therefore, it is becoming more and more popular for tourism research by using the method of analyzing data and excavating the effective information.

The travel route recommendation is mainly based on data mining, location based services (LBSN), GPS trajectory mining technology and popular point recommendation technology have become the most commonly used tourism route recommended auxiliary technology [1-3]. Location-based services become preconditions for route recommendations. Today's travel route planning also uses this technology to make accurate route recommendations so that users can take the shortest route and get the best travel experience. Location information not only creates a new relationship, but more importantly it may make the original relationship more complex. Location maps represent the way in which locations are transferred [4]. The nodes in the figure represent a specific geographical location. The edges between the nodes represent that there are consecutive users accessing the two. The weight of the edge represents the number of consecutive visits, that is, the degree of association between the two points. The travel route recommendation problem is an important field in route recommendation search and recommendation research. Through statistical analysis and excavation of location information shared by users, the user's living habits, interests, 
hobbies, and travel patterns can be obtained. Through the study of the location information in the area, it is possible to obtain the popular route within the area, the locations of people's regular activities, the type of location, and the popularity of the location [5].

Although the existing time-sensitive travel route recommendation method implements route recommendation according to changes in the popularity of sights within one day, it does not take into account that the popularity of many sights and routes is not divided according to a few hours. Moreover, the popularity of various attractions is not the same every day. Just like the two seasons may have the same popular attractions and routes, a season may change over time as popular routes change. The division of time-sensitive time zones is not equal, not limited to the whole month, the whole day, or the entire quarter. It is necessary to take into account the specific trends of popular spots in the year. For this kind of demand, the optimal route for all time divisions in a year is obtained, so as to enhance the user's experience and maximize the user's travel satisfaction.

To address this need, this paper proposes a time-sensitive route recommendation method based on dynamic transition graphs. The route recommendation method is based on the year-round check-in data [6], and a set of transition maps is generated based on the trend of popularity of the sights. The set of transition graphs does not depend on the data of a certain period of time, but dynamically generates a set of transition graphs according to the change in the popularity of all the attractions over time. Thus, the time of one year is divided into a plurality of time periods according to the generation of the dynamic transition graph.

The time-sensitive travel route recommendation method based on the dynamic transition diagram is shown in Fig. 1.

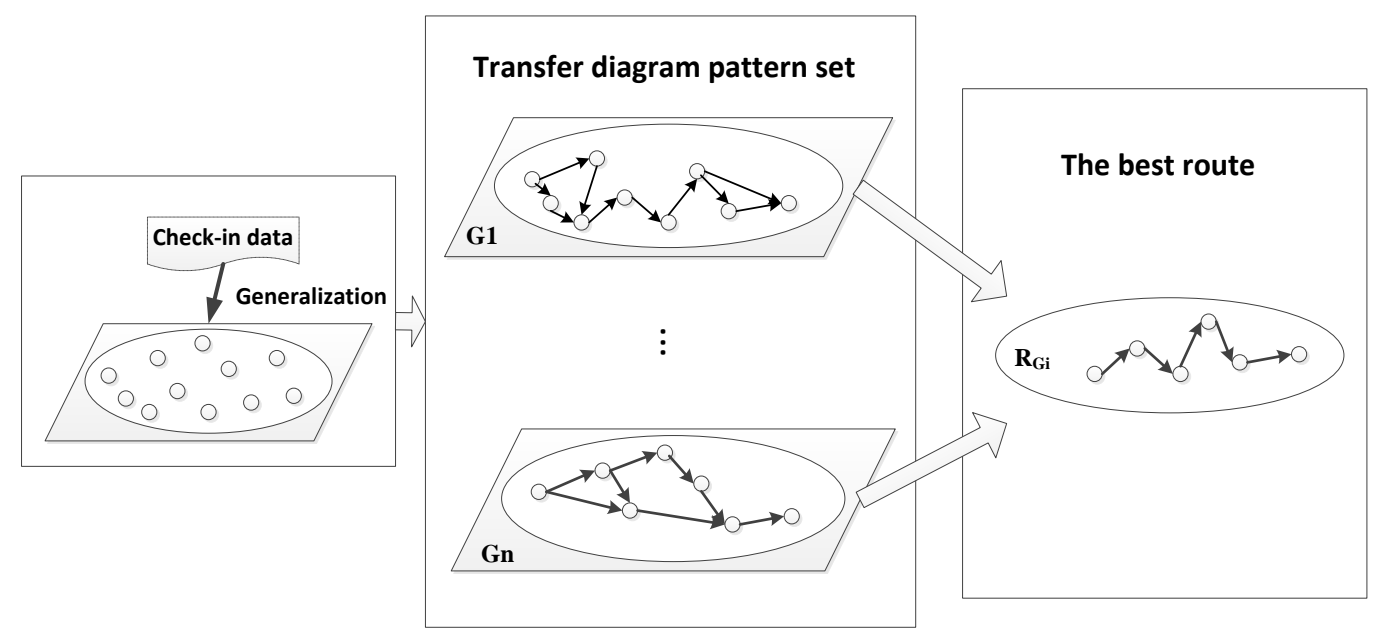

Figure 1. Finite Dynamic Transition Diagram Route Recommendation Model

(1) Preprocessing of data.

This paper uses hierarchical clustering algorithm to generalize. Aggregation of recorded spots in the check-in data was aggregated [7].

(2) Abnormal sequence removal.

In this paper, we use the outlier location method in data mining and mean data replacement method to locate and process the outliers in the sequence [8-10].

(3) The establishment of model sets and route recommendations.

According to the popularity sequence of the attractions, the popular rules and transfer rules of the attractions are drawn, and the rules are learned and divided to obtain a stable and reasonable set of transfer patterns. Combining the time range attributes of the transfer graph pattern set for route recommendation, the time-sensitive travel route recommendation [11-12] is implemented, effectively solving the unique route recommendation problem with different travel times and routes. This paper adopts the method of combining text similarity and hierarchical clustering in data mining to establish a set of patterns. Using transition graph generation rules to establish a set of transition 
graph patterns, and then find the best route for recommendation.

\section{Time-sensitive Route Recommendation Algorithm Based on Dynamic Transition Graph}

Build the model. The process of model construction is shown in Fig. 2, The model is divided into four parts: data pre-processing, point-of-sight model establishment, transfer graph model establishment, and route recommendation. As shown in step 1, this is the preprocessing part of the data. The goal is to remove the deviations that are too finely divided for route recommendations by generalization process. Step 2 is to divide the data according to the number of scenic spots according to the processed data and divide it into equal depth bins to count the frequency of occurrences in each bin. According to the subjective view of real life, time is divided into weeks, so according to the domain knowledge, the popularity of all the attractions can be divided according to the week, and the collection of the attraction patterns and the collection of the popularity of the sights can be constructed. Since each week includes workdays and rest days, the two interfere with the generation of pattern sets. Therefore, the attraction model is divided according to the natural week. And because the collection of the entire year's attractions in the city is extracted and generalized from the flow sequence of numerous objects, the flow sequence is a typical time series. For the time series matching study, the hierarchical clustering method is usually used to establish the mode.

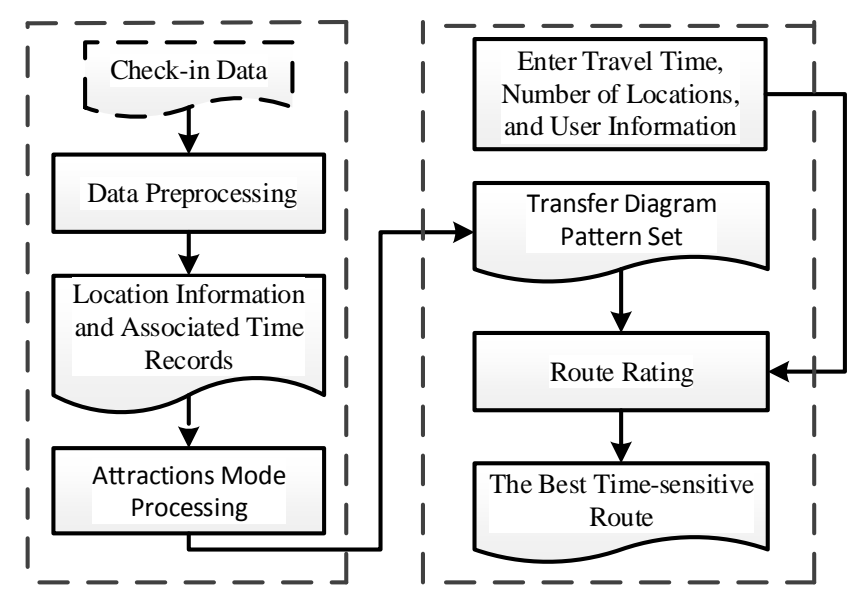

Figure 2. Finite Time-sensitive recommendation model diagram of dynamic transition diagram

The construction and recommendation of the transition graph pattern. The transfer graph is a check-in location with a certain degree of popularity, which excavated from the check-in data shared by a large number of users. Data preprocessing is performed through these check-in locations, and the generalization process is to aggregate the check-in locations and generate the attractions represented by the nodes in the transition graph. When two points of interest have records that the same user has consecutively checked in, a directed edge is generated between both nodes.

The premise of the construction of the transition graph pattern is to remove similar attractions modes. There may be similarities between the attractions modes after the anomaly processing and the similar and adjacent modes may recommend the same or similar routes. Therefore, in this project, the similarity calculation method is used to calculate the similarity between the models. The text similarity adopts the method of hierarchical clustering to aggregate the neighboring patterns in the similarity matrix that meet the aggregation requirements and uses the transfer relationship of the scenic spots in the attractions mode to establish the transition graph pattern. The generation algorithm for the transition graph pattern is as follows.

Algorithm 2.1. Generation of the transition graph pattern

Input: Attractions mode $P$ obtained from $\mathrm{Sn}$;

Output: A set $G$ of the transition graph pattern; 
(1) While $(\operatorname{SimRt} \cdot \max ()>=t h r)$ Do //The maximum value of similarity in the similarity // matrix isnot less thanthe threshold

(2) \{

(3) For the transition graph pattern $P_{k}$ in P Do

$$
\left\{\operatorname{SimRt}=\operatorname{Sim}\left(P_{k}, P_{(k+1)}, k, k+1\right)\right.
$$

$$
\text { max =SimRt.max; //Get the maximum value of the similarity matrix }
$$

Step 1 of Algorithm 2.1 sets the conditions. If the maximum value of the similarity matrix exceeds the threshold in the attractions mode, the aggregation condition is satisfied and the attractions pattern is aggregated. Steps 2-7 are for extracting attraction patterns. Calculate similarity between attractions patterns to generate similarity matrix and aggregate according to hierarchical clustering algorithm. Step 8-9 is to use the aggregated attractions patterns to create transition graphs based on the transition graph generation rules.

The time-sensitive travel route recommendation method based on the dynamic transition graph is to find the transition graph pattern $G$ where the travel time is based on the user's given travel time and the expected number of visited points. Depth-first search strategy is used in $G$ to find all the routes that satisfy the constraint conditions, and the route score is calculated according to the personal preference vector input by the user, and the route of the best revenue is finally recommended for the user. The time-sensitive travel route recommendation method based on the dynamic transition graph is to search for the best route according to the scale of the model, and not to apply the data of the whole year for recommendation as before, so the operating efficiency is greatly improved.

\section{Experiment Analysis}

According to the chart, analyze and compare the Experiments of time-sensitive route recommendation method TRDG based on dynamic transition graph and month-based time-sensitive route recommendation algorithm MTR, quarterly time-sensitive route recommendation algorithm and route recommendation algorithm IPR combined with user's constraints. The advantages of the TRDG algorithm proposed in this paper in accuracy and user satisfaction are obtained. The experiment of this article is carried out from two aspects:

(1) A comparison of the revenue effects of changes in the number of visits to attractions. As the number of attractions changes, the user's overall return value of the route also changes. According to the route benefit calculation method of this paper, the attractions data is directly proportional to the user's profit value. The more the number of tourist attractions set by the user, the higher the user's return value will be. But in the case of the same number of attractions, it is possible to compare algorithm;

(2) A comparison of the revenue effects of different travel times. The popularity of attractions changes over time. Different travel times for the same attractions may give users different profit values. With the same travel time, the pros and cons of the algorithm can be compared;

Fig. 3 shows Profit's change with the number $\mathrm{N}$ of visit locations under the condition that the user has the same preference and travels in March 1. The profit of each algorithm increases with the number $\mathrm{N}$ of visit locations, indicating that the larger the number of places, the higher the user's profit. Among them, STR and IPR recommendations are poor, MTR is slightly better, and TRDG's Profit is about $15 \%$ higher than the other three algorithms. 


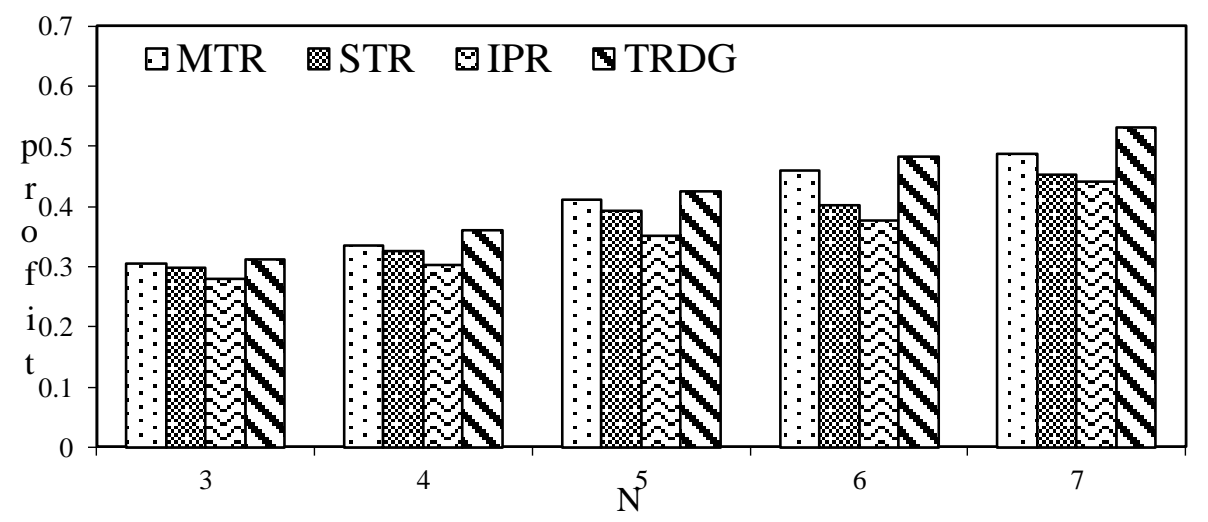

Figure 3. Finite Number of visits $\mathrm{N}$ impact on Profit

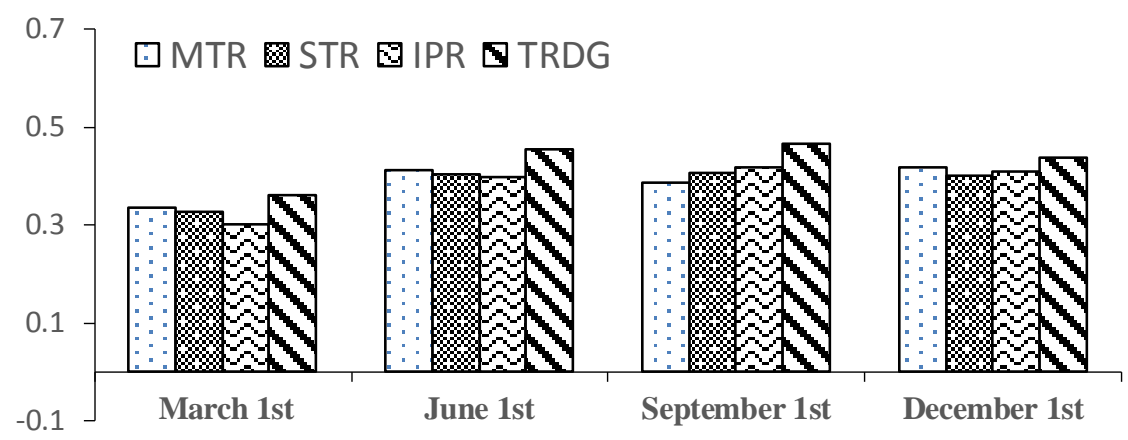

Figure 4. Travel time t impact on Profit

Fig. 4 is a comparison of user's profit for different travel times using four algorithms. Among them, Profit is under the condition that the number of visits to the same user is 4, travel times are in four different months and different seasons, such as, March 1st, June 1st, September 1st, and December 1st. The user's profit of TRDG is at least $15 \%$ higher than that of the other three route recommendation algorithms.

\section{Conclusions}

This paper proposes a new route recommendation method which called time-sensitive travel route recommendation based on dynamic transition graph. It quantitatively expresses changes in the popularity of the attractions in the form of a sequence and establishes a sights mode through abnormal processing of sequences. According to the calculation of the similarity of the attractions pattern, similar patterns are aggregated to generate a set of dynamic attractions patterns in the area. Our specific tasks include: Discovery of regional popular attractions; establishment of attractions popularity sequences; construction of attractions models; construction of transfer graph models; time-sensitive route recommendations.

The proposed method of time-sensitive travel route recommendation based on dynamic transition graph is fully validated using real data sets. It is proved that the proposed exception handling method for popularity sequences and the similarity calculation method of the attractions pattern all play an important role in the pattern generation. The experiment analyzes and compares the effect of the algorithm and the user's benefits and verifies the correctness of the research and the superiority of the algorithm. 


\section{References}

[1] Bao J, Zheng Y, Wilkie D, et al. Recommendations in location-based social networks: a survey [J]. GeoInformatica, 2015, 19(3): 525-565.

[2] Bao J, Zheng Y, Mokbel M F. Location-based and preference-aware recommendation using sparse geo-social networking data[C]//Proceedings of the 20th International Conference on Advances in Geographic Information Systems. ACM, 2012: 199-208.

[3] Funke S, Storandt S. Personalized route planning in road networks[C]//Proceedings of the 23rd SIGSPATIAL International Conference on Advances in Geographic Information Systems. ACM, 2015: 45.

[4] Hsieh H P, Li C T. Composing Traveling Paths from Location-Based Services[C]//ICWSM. 2012.

[5] Qingxia Wu, Ya Zhou, Diyao Wen, et al. Personalized travel route recommendations based on user interest and popularity of points of interest[J]. Computer Application, 2016, 36(6).

[6] Hsieh H P, Li C T, Lin S D. Exploiting large-scale check-in data to recommend time-sensitive routes[C]//Proceedings of the ACM SIGKDD International Workshop on Urban Computing. ACM, 2012: 55-62..

[7] Hsieh H P, Li C T. Constructing trip routes with user preference from location check-in data[C]/Proceedings of the 2013 ACM conference on Pervasive and ubiquitous computing adjunct publication. ACM, 2013: 195-198.Zheng Y, Xie X. Learning travel recommendations from user-generated GPS traces [J]. ACM Transactions on Intelligent Systems and Technology (TIST), 2011, 2(1): 2.

[8] Zheng Y. Tutorial on location-based social networks[C]//Proceedings of the 21st international conference on World wide web. 2012.

[9] Zheng Y, Zhang L, Xie X, et al. Mining interesting locations and travel sequences from GPS trajectories[C]//Proceedings of the 18th international conference on World wide web. ACM, 2009: 791-800.

[10] Xiaoyu Song, Hongfei Xu, Huanliang Sun, et al. Short-time experiential route search based on check-in data [J]. Journal of Computer, 2013, 36(8): 1693-1703.

[11]Dai J, Yang B, Guo C, et al. Personalized route recommendation using big trajectory data[C]//Data Engineering (ICDE), 2015 IEEE 31st International Conference on. IEEE, 2015: 543-554.

[12] Ye $\mathrm{M}$, Yin $\mathrm{P}$, Lee $\mathrm{W} \mathrm{C}$, et al. Exploiting geographical influence for collaborative point-of-interest recommendation[C]//Proceedings of the 34th international ACM SIGIR conference on Research and development in Information Retrieval. ACM, 2011: 325-334. 